\title{
Incidences of Unfavorable Events in the Management of Low-Risk Papillary Microcarcinoma of the Thyroid by Active Surveillance Versus Immediate Surgery
}

\author{
Hitomi Oda, ${ }^{1}$ Akira Miyauchi, ${ }^{1}$ Yasuhiro Ito, ${ }^{1,2}$ Kana Yoshioka, ${ }^{3}$ Ayako Nakayama, ${ }^{3}$ \\ Hisanori Sasai, ${ }^{3}$ Hiroo Masuoka, ${ }^{1}$ Tomonori Yabuta, ${ }^{1}$ Mitsuhiro Fukushima, ${ }^{1}$ Takuya Higashiyama, \\ Minoru Kihara, Kaoru Kobayashi, and Akihiro Miya ${ }^{1}$
}

Background: The incidence of papillary microcarcinoma (PMC) of the thyroid is rapidly increasing globally, making the management of PMC an important clinical issue. Excellent oncological outcomes of active surveillance for low-risk PMC have been reported previously. Here, unfavorable events following active surveillance and surgical treatment for PMC were studied.

Methods: From February 2005 to August 2013, 2153 patients were diagnosed with low-risk PMC. Of these, 1179 patients chose active surveillance and 974 patients chose immediate surgery. The oncological outcomes and the incidences of unfavorable events of these groups were analyzed.

Results: In the active surveillance group, 94 patients underwent surgery for various reasons; tumor enlargement and the appearance of novel lymph node metastases were the reasons in $27(2.3 \%)$ and six patients $(0.5 \%)$, respectively. One of the patients with conversion to surgery had nodal recurrence, and five patients in the immediate surgery group had a recurrence in a cervical node or unresected thyroid lobe. All of these recurrences were successfully treated. None of the patients had distant metastases, and none died of the disease. The immediate surgery group had significantly higher incidences of transient vocal cord paralysis (VCP), transient hypoparathyroidism, and permanent hypoparathyroidism than the active-surveillance group did (4.1\% vs. $0.6 \%$, $p<0.0001 ; 16.7 \%$ vs. $2.8 \%, p<0.0001$; and $1.6 \%$ vs. $0.08 \%, p<0.0001$, respectively). Permanent VCP occurred only in two patients $(0.2 \%)$ in the immediate surgery group. The proportion of patients on L-thyroxine for supplemental or thyrotropin (TSH)-suppressive purposes was significantly larger in the immediate surgery group than in the active surveillance group $(66.1 \%$ vs. $20.7 \%, p<0.0001)$. The immediate surgery group had significantly higher incidences of postsurgical hematoma and surgical scar in the neck compared with the active surveillance group ( $0.5 \%$ vs. $0 \%, p<0.05$; and $8.0 \%$ vs. $100 \%, p<0.0001$, respectively).

Conclusions: The oncological outcomes of the immediate surgery and active surveillance groups were similarly excellent, but the incidences of unfavorable events were definitely higher in the immediate surgery group. Thus, active surveillance is now recommended as the best choice for patients with low-risk PMC.

\section{Introduction}

$\mathbf{P}$ APILLARY THYROID CARCINOMA (PTC) is the most common type of thyroid cancer, accounting for approximately $90 \%$ of all thyroid malignancies. In 2014, Davies and Welch reported a rapid increase in the incidence of thyroid cancer in the United States, with a 2.9 -fold increase over the past 35 years (1). The increase was due to the increase in PTCs of only small size. The proportion of tumors $\leq 1 \mathrm{~cm}$ was reported to reach $39 \%$ of all PTCs, whereas the incidences of other types of thyroid cancer have remained stable. A PTC $\leq 1 \mathrm{~cm}$ in maximum diameter is called a papillary microcarcinoma (PMC) of the thyroid (2). Most importantly, the mortality from thyroid cancer has remained stable.

The above data strongly suggest that the increase in the incidence of thyroid cancer is mainly due to the increase in the detection of small PTCs because of the increasing use of many imaging studies. In Korea, the incidence of thyroid cancer attained a 15 -fold increase (3), and this increase was also due to an increase in small PTCs; the thyroid cancer

\footnotetext{
${ }^{1}$ Department of Surgery; ${ }^{2}$ Clinical Trial Management Center; ${ }^{3}$ Department of Head and Neck Surgery; Kuma Hospital, Kobe, Japan.
}

(C) Oda et al. 2015; Published by Mary Ann Liebert, Inc. This Open Access article is distributed under the terms of the Creative Commons Attribution Noncommercial License (http://creativecommons.org/licenses/by-nc/4.0/) which permits any noncommercial use, distribution, and reproduction in any medium, provided the original author(s) and the source are credited. 
mortality rate in Korea has also remained stable. This scenario has become a global phenomenon in well-developed countries. A similar trend started much earlier in Japan, probably because the use of ultrasound examinations and fine-needle aspiration biopsy (FNAB) for the detection and evaluation of thyroid nodules was started much earlier than in the United States and Korea.

Takebe et al. conducted a thyroid cancer screening study using ultrasound examinations and ultrasound-guided FNABs at the time of screening studies for breast cancer, and they found that $3.5 \%$ of the otherwise healthy adult Japanese women they screened had small thyroid cancers (4). This incidence was more than 1000 times the reported prevalence of clinical thyroid cancer in Japanese women at that time. Many autopsy studies have also reported high incidences of latent thyroid cancer in subjects who died of nonthyroidal causes up to $5.2 \%$ for tumors $>3 \mathrm{~mm}$ (5-8), which can be detected and diagnosed with ultrasound-guided FNAB. The incidence reported by Takebe $e t a l$. is thus consistent with the reported incidence of latent thyroid cancer in autopsy studies, raising a serious question about the necessity of treatment for incidentally detected asymptomatic small PTCs.

Miyauchi hypothesized that most PMCs stay small and do not cause any harm to the hosts, and that only a few PMCs grow to clinical cancer that could cause harm to the hosts. He also speculated that performing surgery only for PMCs that show progression might not be too late. He proposed an observational clinical trial for low-risk PMCs at a physician meeting of Kuma Hospital in 1993, which was approved. In 1993, physicians at Kuma Hospital started an active surveillance clinical trial for patients with low-risk PMCs who were examined at Kuma Hospital. Patients were offered the choice of two options: observation without surgery and immediate surgery. A similar observation trial was started two years later at the Cancer Institute Hospital in Tokyo. The early results of these two trials were very similar and assuring. In 2014, the updated outcomes of the active surveillance trial were reported: the cumulative ratio of the patients whose PMC increased by $\geq 3 \mathrm{~mm}$ was $8 \%$ at 10 years, and the cumulative ratio of the patients who showed novel lymph node metastasis was $3.8 \%$ at 10 years (9). None of the patients developed distant metastases, and none died of thyroid cancer. These oncological outcomes are assuring. However, any management or treatment of a disease might be associated with negative features. Therefore, the present study compared the incidences of unfavorable events such as vocal cord paralysis, hypoparathyroidism, need for L-thyroxine, and others in PMC patients who chose active surveillance and those who chose immediate surgery.

\section{Patients and Methods}

From February 2005 to August 2013 at Kuma Hospital, 2153 patients were diagnosed with low-risk PMC by ultrasound-guided FNAB, excluding those cases associated with Graves' disease or primary hyperparathyroidism and those with a follow-up period shorter than one year. The patients included 1894 females and 259 males aged $15-88$ years (median 56 years). Patients diagnosed with PTC were evaluated for the presence or absence of aggressive features such as nodal or distant metastasis, macroscopic extrathyroidal extension, high-grade malignancy on cytology, and evidence of progression and for worrisome features such as tumors attached to the trachea or located on the course of the recurrent laryngeal nerve. Patients with one or more of these aggressive or worrisome features were regarded as high risk, and immediate surgery was recommended for them. The patients with none of these aggressive or worrisome features were regarded as low risk and were enrolled in the present study.

When a patient was diagnosed with low-risk PMC, the patient was presented with the choice of two management options: active surveillance or immediate surgery. Of the 2153 low-risk PMC patients, 1179 patients (54.8\%) chose active surveillance, and 974 patients $(45.2 \%)$ chose immediate surgery. The background clinical characteristics of these patients are shown in Table 1. The groups had similar sex distributions, with approximately $88 \%$ female patients, similar study periods (12-116 months, median 47 months), incidences of hypothyroidism (3.1-4.3\%), family history of thyroid cancer (3.1-3.5\%), and association of benign thyroid nodules $>20 \mathrm{~mm}(17.0-17.9 \%)$. However, compared with the active surveillance group, the immediate surgery group was slightly younger (median 55 years vs. 57 years), and had slightly larger tumors (median $8 \mathrm{~mm}$ vs. $7 \mathrm{~mm}$ ), more multiple tumors (13.6\% vs. $6.0 \%)$, and a lower incidence of positive test results for thyroid antibodies $(26.7 \%$ vs. $30.8 \%)$.

The patients who chose observation were followed with ultrasound examinations and thyroid function tests, including

Table 1. Clinical Characteristics of Low-Risk PMC Patients Who Chose Active Surveillance AND Those Who Chose IMMediate Surgery

\begin{tabular}{lccc}
\hline & \multicolumn{2}{c}{ Intended management } & \\
\cline { 2 - 3 } Clinical characteristics & Active surveillance & Immediate surgery & Significance \\
\hline Male/female & $142 / 1037$ & $117 / 857$ & n.s. \\
Age (years) & $57(15-88)$ & $55(15-84)$ & $p<0.0001$ \\
Tumor size $(\mathrm{mm})^{\mathrm{a}}$ & $7(2-10)$ & $8(3-10)$ & $p<0.0001$ \\
Study period $(\mathrm{months})^{\mathrm{a}}$ & $47(12-116)$ & $47(12-116)$ & n.s. \\
Multiplicity & $71 / 1179(6.0 \%)$ & $132 / 974(13.6 \%)$ & $p<0.0001$ \\
Hypothyroidism & $51 / 1179(4.3 \%)$ & $30 / 974(3.1 \%)$ & n.s. \\
TgAb (+) or TPOAb $(+)$ & $363 / 1179(30.8 \%)$ & $260 / 974(26.7 \%)$ & $p<0.01$ \\
Familial history: yes & $36 / 1179(3.1 \%)$ & $34 / 974(3.5 \%)$ & n.s. \\
Benign thyroid nodule $(>20 \mathrm{~mm}):$ yes & $200 / 1179(17.0 \%)$ & $174 / 974(17.9 \%)$ & n.s. \\
\hline
\end{tabular}

${ }^{a}$ Median value (range).

PMC, papillary microcarcinoma; TgAb, thyroglobulin antibodies; TPOAb, thyroid peroxidase antibodies; n.s., not significant. 
serum calcium, six months after the diagnosis and once a year thereafter to check for possible changes in the tumor size and the appearance of novel lymph node metastases. Patients who had changes in their voice underwent fiber-optic laryngoscopy. The observation period ranged from 12 to 116 months with a median of 47 months.

The patients who chose immediate surgery underwent the procedure within 11 months (median 2 months) of the diagnosis. The surgery was hemithyroidectomy and paratracheal dissection in 575 patients, and total thyroidectomy with central compartment neck dissection in 399 patients. Fiberoptic laryngoscopy was routinely performed preoperatively, immediately after the extubation of a tracheal tube, and two days after surgery. For the patients who underwent a total thyroidectomy, serum calcium and intact parathyroid hormone (i-PTH) were routinely measured in the morning on the day after surgery. All of the immediate surgery patients were followed with periodic ultrasound examinations of the neck and thyroid function tests, as well as parathyroid function tests when indicated. L-thyroxine was prescribed to maintain the serum thyrotropin (TSH) level at around the lower normal range, and alfacalcidol and calcium lactate were prescribed if necessary. Patients who required alfacalcidol for more than one year were regarded as having permanent hypoparathyroidism. The patients were followed after surgery for a median of 47 months (range 12-116 months).

Statistical analyses to determine any significant differences between the active surveillance and immediate surgery groups were performed using the chi-square test for nominal variables and the Mann-Whitney $U$-test for continuous variables. $p$-Values $<0.05$ were accepted as significant.

\section{Results}

\section{Oncological outcome}

Of the 1179 patients who chose active surveillance, 94 patients $(8.0 \%)$ underwent surgery after a period of observation longer than one year (Fig. 1). The main reason was that the patient simply changed his or her mind (51 patients, $54 \%$ of 94

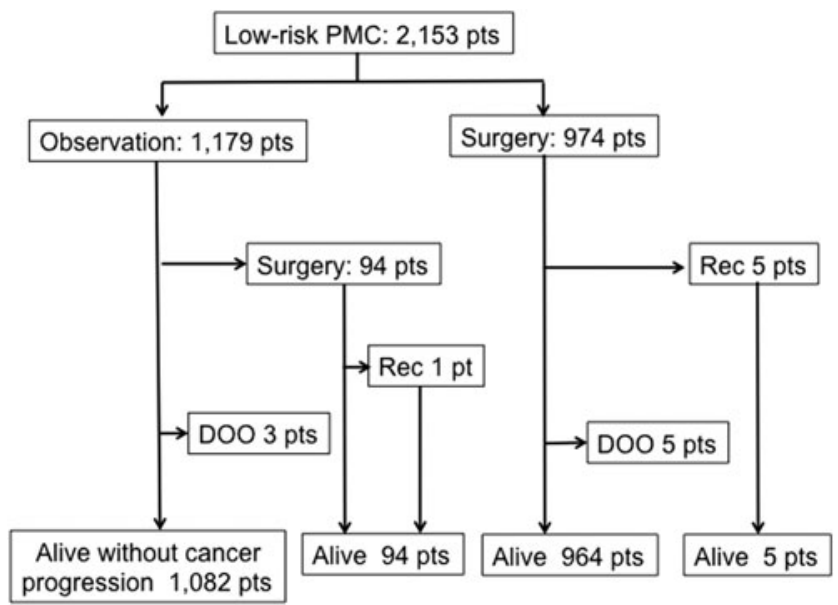

FIG. 1. Flow of the management and oncological outcomes of 2153 patients with low-risk papillary microcarcinoma (PMC). Of the observation group, 94 patients underwent surgery for various reasons. Rec: recurrence; DOO, died of other causes unrelated to thyroid cancer. patients with conversion), whereas the tumor showed an increase in size by $\geq 3 \mathrm{~mm}$ in 27 patients (2.3\% of 1179 patients), and novel lymph node metastases appeared in six patients ( $0.5 \%$ of 1179 patients). Enlargement of associated benign nodules was the reason in five patients. The reason was unclear in the remaining five patients. One of the patients who underwent a conversion surgery developed recurrence in the lateral neck compartment, which was treated successfully with a modified neck dissection.

Of the 974 patients who underwent immediately surgery, five patients developed recurrence (Fig. 1). Three of the five patients showed the appearance of the tumor in the contralateral lobe that was not resected at the initial surgery. Completion thyroidectomy was performed in these patients. Two other patients developed lymph node metastases in the lateral neck compartment; one of them underwent a modified neck dissection. The other patient is under watchful observation with mild TSH suppressive therapy, since the metastatic nodes were small in size.

Three patients in the observation group and five patients in the immediate surgery group died of diseases unrelated to thyroid cancer. None of the present study's patients developed distant metastases, and none died of thyroid cancer. Thus, none of the patients of either group had an unfavorable oncological outcome.

\section{Incidence of vocal cord paralysis}

The immediate surgery group had significantly higher incidence of temporary vocal cord paralysis (VCP) than the active surveillance group did (4.1\% vs. $0.6, p<0.0001$; Table 2$)$. Six cases of VCP in the active surveillance group occurred among the patients with conversion surgery, and the remaining case had a temporary idiopathic VCP that occurred contralateral to the PMC. Permanent VCP occurred only in two patients $(0.2 \%)$ in the immediate surgery group. The recurrent laryngeal nerve was accidentally transected during the initial surgery in one of these two patients; the nerve ends were anastomosed during the same surgery. Another patient had her recurrent laryngeal nerve ligated accidentally together with a vessel. The ligation thread was removed shortly after the accident. These patients' voices recovered, but the vocal cords on the affected side remained immobile at the median. Thus, all of the cases of temporary and permanent VCP occurred due to thyroid surgery, except for one case of temporary idiopathic VCP.

\section{Incidence of hypoparathyroidism}

The immediate surgery group had a significantly higher incidence of temporary hypoparathyroidism compared with the active surveillance group $(16.7 \%$ vs. $2.8 \%, p<0.0001$; Table 2). All of the cases of temporary hypoparathyroidism that were seen in the active surveillance group occurred in the patients who underwent conversion surgery. The incidence of permanent hypoparathyroidism was also significantly higher in the immediate surgery group than it was in the active surveillance group $(1.6 \%$ vs. $0.08 \%, p<0.0001)$. One of the patients in the active surveillance group developed idiopathic hypoparathyroidism during the study period. Thus, all of the cases of transient and permanent hypoparathyroidism resulted from thyroid surgery, except for the single case of idiopathic hypoparathyroidism. 
Table 2. Unfavorable Events Following Active Surveillance and Immediate Surgery

\begin{tabular}{lccc}
\hline & \multicolumn{3}{c}{ Intended management } \\
\cline { 2 - 3 } Unfavorable events & $\begin{array}{c}\text { Active surveillance, } \\
1179 \text { pts }\end{array}$ & $\begin{array}{c}\text { Immediate surgery, } \\
974 \text { pts }\end{array}$ & p-Value \\
\hline Later surgery (pts) & 94 & 0 & $<0.0001$ \\
Temporary VCP (\%) & $7(0.6 \%)$ & $40(4.1 \%)$ & $<0.0001$ \\
Permanent VCP (\%) & $0(0 \%)$ & $2(0.2 \%)$ & n.s. \\
Temporary Hypo-PT (\%) & $33(2.8 \%)$ & $163(16.7 \%)$ & $<0.0001$ \\
Permanent Hypo-PT (\%) & $1(0.08 \%)$ & $16(1.6 \%)$ & $<0.0001$ \\
On L-thyroxine (\%) & $244(20.7 \%)$ & $544(66.1 \%)$ & $<0.0001$ \\
Postsurgical hematoma (\%) & $0(0 \%)$ & $0.5 \%)$ & n.s. \\
Postsurgical abscess $(\%)$ & $0(0 \%)$ & $974(100 \%)$ & $<0.0001$ \\
Surgical scar (\%) & $94(8.0 \%)$ & 5 & n.s. \\
Recurrence in neck (pts) & 1 & $5(0.5 \%)$ & n.s. \\
Death (\%) & $3(0.3 \%)$ & & \\
\hline
\end{tabular}

VCP and Hypo-PT in the active surveillance group occurred in patients who converted to surgery later for various reasons, except for one patient who developed idiopathic Hypo-PT and another who developed transient idiopathic VCP contralateral to the microcarcinoma. All deaths in the present series were due to causes unrelated to thyroid cancer.

VCP, vocal cord paralysis; Hypo-PT, hypoparathyroidism.

\section{Proportion of patients on L-thyroxine therapy}

All of the patients who underwent a total thyroidectomy inevitably required L-thyroxine. L-thyroxine was also prescribed for the patients who had undergone a hemithyroidectomy as supplemental therapy or as mild TSH suppressive therapy. The cases of some of the patients in the active surveillance group were associated with hypothyroidism requiring L-thyroxine, and others were prescribed the medicine as mild TSH suppressive therapy. The proportion of patients on L-thyroxine was significantly higher in the immediate surgery group than in the active surveillance group $(66.1 \%$ vs. $20.7 \%, p<0.0001$; Table 2 ).

\section{Incidences of other unfavorable events}

The immediate surgery group had significantly higher incidences of postsurgical hematomas requiring treatment, and all had a surgical scar in the neck compared with the active surveillance group $(0.5 \%$ vs. $0 \%, p<0.05$; and $100 \%$ vs. $8.0 \%, p<0.0001$, respectively; Table 2). All surgical scars in the active surveillance group were due to conversion to surgery.

\section{Discussion}

Because of the increasing prevalence of imaging studies, asymptomatic small PTCs are being increasingly detected incidentally, and they can be easily diagnosed with ultrasoundguided FNAB $(1,10)$. Even PMCs $\leq 1 \mathrm{~cm}$ in maximum diameter may be associated with lymph node metastases, distant metastases (although very rare), or invasion to the recurrent laryngeal nerve causing VCP. These symptomatic PMCs have aggressive characteristics and should be treated appropriately. However, the vast majority of the PMCs incidentally detected during imaging studies do not show these aggressive features.

It was hypothesized that most asymptomatic PMCs stay small and do not cause any harm to the hosts, and an active surveillance clinical trial for low-risk PMC was initiated in 1993. Two years later, a similar trial was started at the Cancer Institute Hospital in Tokyo. In 2003, the early results of the trial were reported. Only 162/732 (22\%) patients with lowrisk PMC chose observation (11). At five years of the observation, $27.5 \%$ of the tumors showed an increase in size by $\geq 2 \mathrm{~mm}, 60.3 \%$ stayed the same size, and $12.1 \%$ showed a decrease in size. Only $1.2 \%$ of the patients showed the appearance of novel nodal metastases, which were treated successfully. Sugitani et al. at the Tokyo Cancer Institute Hospital reported that in their nonsurgical observation study for a five-year period of 230 patients with PMCs, only $7 \%$ of the PMCs showed an increase in size, and $1 \%$ of the patients showed the appearance of nodal metastases that were treated successfully; none of their patients died of thyroid cancer (12).

With these promising study results, the Clinical Management Guidelines of Thyroid Tumors 2010 were published by the Japanese Association of Endocrine Surgeons, and the Japanese Society of Thyroid Surgery accepted nonsurgical observation as a management option for low-risk PMC. These became the first guidelines that accepted an active surveillance policy.

In 2014, the results of the active surveillance trial of 1235 patients were updated. The cumulative ratio of patients whose PMC increased by $\geq 3 \mathrm{~mm}$ was $8 \%$ at 10 years, and the cumulative ratio of patients who showed novel lymph node metastasis at 10 years was $3.8 \%$ (9). Patients with these progressions were successfully treated surgically. This study clarified that the progression of PMC was strongly associated with young age $(<40$ years) of the patients. In contrast to advanced PTC, PMCs in elderly patients ( $>60$ years) tended to stay small without showing nodal metastasis. None of the patients developed distant metastases, and none died of thyroid cancer. These oncological outcomes of nonsurgical observation for low-risk PMCs were assuring. The present study also shows excellent oncological outcomes of active surveillance comparable to those of the immediate surgery.

However, any treatment or management of a disease can be associated with negative or unfavorable features. For thyroid cancer, surgical resection is generally the first-line treatment, but thyroid surgery may cause vocal cord paralysis, hypoparathyroidism, postoperative hematoma, and/or the necessity for supplemental L-thyroxine, and a surgical scar is 
inevitable. The results of the present study revealed that the immediate surgery group had significantly higher incidences of transient VCP, transient hypoparathyroidism, and permanent hypoparathyroidism than the active surveillance group did. The comparison was made between the two patient groups based on the intention to treat basis. All of these unfavorable events occurred in patients who had thyroid surgery as the initial treatment or as a conversion from surveillance, except for one case of transient idiopathic VCP and one case of idiopathic hypoparathyroidism that occurred during the observation.

None of the active surveillance patients developed VCP because of PMC. Unfortunately, 2/974 patients who underwent immediate surgery showed permanent VCP due to accidental injury of the recurrent laryngeal nerve, and although this incidence was low at $0.2 \%$, the consequence was serious, and this occurred solely in patients who underwent thyroid surgery. The transected nerve ends were anastomosed during the same surgery, and the patients' voices recovered to nearly normal. The vocal cords on the same side, however, remained immobile, as reported previously (13).

All of the surgeries in the present study were performed by surgeons at the Center for Excellence in Thyroid Care at Kuma Hospital. Over the course of the study period, 10 endocrine surgeons and two ear, nose, and throat (ENT) physicians performed the surgeries, of whom all but two trainees were well experienced and high-volume surgeons. The average blood loss and operation times for hemithyroidectomy with paratracheal dissection and total thyroidectomy with central compartment dissection were $15 \mathrm{~g}$ and $69 \mathrm{~min}$, and $29 \mathrm{~g}$ and $103 \mathrm{~min}$, respectively, illustrating the surgeons' excellent surgical skills. However, surgical complications occurred even at this level of skill and experience. The surgeries for PMCs are simple, but nevertheless human errors can occur. Prophylactic central compartment dissection was performed for the total thyroidectomy cases and prophylactic paratracheal dissection for the hemithyroidectomy cases in order to avoid a reoperation of the central neck in case of a recurrence. If the extent of surgery was more limited, the incidence of complications might have been less but would still be present.

The surgical complication rates among less experienced and low-volume surgeons were reported to be higher than those in well experienced and high-volume surgeons (14). If less experienced surgeons had performed the surgeries in the present study, the complication rates would have been much higher. One of the present authors (A.M.) treated a female patient who exhibited bilateral VCP immediately after hemithyroidectomy with central compartment dissection for PMC that was performed at one of the main hospitals in a different prefecture in Japan. Several years later, she required a tracheostomy to relieve dyspnea after she caught a cold. Her bilateral VCP would never have occurred if she had not undergone the surgery.

The unfavorable events in the present immediate surgery group also included significantly higher incidences of the prescription of L-thyroxine, postsurgical hematomas, and, inevitably, surgical scars in the neck compared with the active surveillance group. Although these might be less important compared with VCP or hypoparathyroidism, these events certainly negatively affect the patients' quality of life. Other possible unfavorable issues are a choking feeling in the neck that some patients experience after thyroid surgery, and the costs of the case management. The authors are currently preparing a manuscript on the latter issue, and it can already be concluded that surgery is much more expensive compared with observation.

Another issue is the potential for patients to experience fear or anxiety at the thought of leaving their PMCs untreated. A complete explanation and education are very important to relieve patients' fear, and that providing information and education on this issue to physicians who take care of such patients is also very important. Most patients understand which is the better choice when they learn about the actual outcomes of immediate surgery and active surveillance. At an early period of the observation clinical trial, only $22 \%$ of the patients with low-risk PMC chose observation (11). During the present study period of 2005-2013, $54.8 \%$ of the candidates chose observation. Patients' responses and choices depended strongly on who explained the options and how the options were explained. With the results of the present study, there is growing confidence that active surveillance is superior to immediate surgery for low-risk PMC. Although the two options are still offered to PMC patients, active surveillance is now strongly recommended as the best choice for patients with low-risk PMC.

In conclusion, the oncological outcomes of immediate surgery and active surveillance were similarly excellent. The incidences of unfavorable events were definitely higher in the immediate surgery group than in the active surveillance group, and now active surveillance is recommended as the best choice for patients with low-risk PMCs.

\section{Author Disclosure Statement}

The authors state that there are no conflicts of interest related to this study.

\section{References}

1. Davies L, Welch HG 2014 Current thyroid cancer trends in the United States. JAMA Otolaryngol Head Neck Surg 140:317-322.

2. Sobin LH 1990 Histological typing of thyroid tumours. Histopathology 16:513.

3. Ahn HS, Kim HJ, Welch HG 2014 Korea's thyroid-cancer "epidemic" - screening and overdiagnosis. N Engl J Med 371:1765-1767.

4. Takebe K, Date M, Yamamoto Y 1994 Mass screening for thyroid cancer with ultrasonography. KARKINOS 7:309317 [in Japanese].

5. Harach HR, Franssila KO, Wasenius VM 1985 Occult papillary carcinoma of the thyroid. A "normal" finding in Finland. A systematic autopsy study. Cancer 56:531-538.

6. Fukunaga FH, Yatani R 1975 Geographic pathology of occult thyroid carcinomas. Cancer 36:1095-1099.

7. Samson RJ 1977 Prevalence and significant of occult thyroid cancer. In: DeGroot LJ (ed) Radiation-Associated Thyroid Carcinoma. Grune \& Stratton, New York, NY, pp $137-153$.

8. Thorvaldsson SE, Tulinius $\mathrm{H}$, Björnsson J, Bjarnason $\mathrm{O}$ 1992 Latent thyroid carcinoma in Iceland at autopsy. Pathol Res Pract 188:747-750.

9. Ito Y, Miyauchi A, Kihara M, Higashiyama T, Kobayashi K, Miya A 2014 Patient age is significantly related to the 
progression of papillary microcarcinoma of the thyroid under observation. Thyroid 24:27-34.

10. Brito JP, Morris JC, Montori VM 2013 Thyroid cancer: zealous imaging has increased detection and treatment of low risk tumours. BMJ 347:f4706.

11. Ito $\mathrm{Y}$, Uruno $\mathrm{T}$, Nakano $\mathrm{K}$, Takamura $\mathrm{Y}$, Miya A, Kobayashi K, Yokozawa T, Matsuzuka F, Kuma S, Kuma K, Miyauchi A 2003 An observation trial without surgical treatment in patients with papillary microcarcinoma of the thyroid. Thyroid 13:381-387.

12. Sugitani I, Toda K, Yamada K, Yamamoto N, Ikenaga M, Fujimoto Y 2010 Three distinctly different kinds of papillary thyroid microcarcinoma should be recognized: our treatment strategies and outcomes. World J Surg 34:12221231.

13. Miyauchi A, Inoue H, Tomoda C, Fukushima M, Kihara M, Higashiyama, Takamura Y, Ito Y, Kobayashi K, Miya A 2009 Improvement in phonation following reconstruction of the recurrent laryngeal nerve in patients with thyroid cancer invading the nerve. Surgery 146:1056-1062.

14. Hauch A, Al-Qurayshi Z, Randolph G, Kandil E 2014 Total thyroidectomy is associated with increased risk of complications for low- and high-volume surgeons. Ann Surg Oncol 21:3844-3852.

Address correspondence to: Akira Miyauchi, $M D, P h D$ Department of Surgery Kuma Hospital 8-2-35 Shimoyamate-dori Chио-Ku Kobe 650-0011 Japan

E-mail: miyauchi@kuma-h.or.jp 